\title{
Efficacy of chili plant diseases classification using deep learning: a preliminary study
}

\author{
Suhana Rozlan, Marsyita Hanafi \\ Department of Computer and Communication Systems Engineering, Faculty of Engineering, Universiti Putra Malaysia, Selangor, \\ Malaysia
}

\begin{tabular}{l} 
Article Info \\
\hline Article history: \\
Received Aug 26, 2021 \\
Revised Dec 7, 2021 \\
Accepted Jan 12, 2022 \\
\hline
\end{tabular}

Keywords:

Chili leave diseases

Complex images

Deep learning

Plant disease classification

Transfer learning

\begin{abstract}
Plant disease classification using deep learning techniques is a popular research area due to the numerous opportunities for introducing advance and robust classifiers. Nevertheless, classifying chilli plant diseases accurately from images under uncontrolled environment and various imaging conditions remains unsolved due to the lack of chilli disease image datasets. In this study, the efficacy of three high-performance deep learning algorithms, namely VGG16, InceptionV3, and EfficientNetB0, in classifying three types of chilli leaves diseases, namely upward curling, mosaic/mottling, and the bacterial spot, is demonstrated. These methods are popularly used for other plant disease classifications due to their effectiveness. The experiments were performed on the 3,000 chilli plant disease images collected from three different field environments in Selangor, Malaysia. The images were captured with a complex background and various illuminations, angles, and distances to reflect the real-life scenarios. The complexity of the collected images was created based on the taxonomic information of chilli leaves diseases and the unavailability of chilli disease images under various imaging conditions in the publicly available plant disease databases. Experimented using appropriate specifications, the models demonstrated outstanding performance with more than 95\% accuracy with the highest accuracy of $98.83 \%$ by InceptionV3.
\end{abstract}

This is an open access article under the $\underline{C C B Y-S A}$ license.

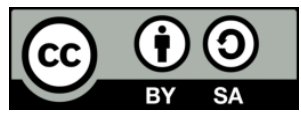

\section{Corresponding Author:}

Marsyita Hanafi

Department of Computer and Communication Systems Engineering, Faculty of Engineering

Universiti Putra Malaysia

43400 UPM Serdang, Selangor, Malaysia

Email: marsyita@upm.edu.my

\section{INTRODUCTION}

Chilli or scientifically known as capsicum annuum L. is Malaysia's utmost cultivated crop [1] and has been recognized as among the top tenth of self-sufficiency ratio (SSR) in selected agricultural commodities with the highest import dependency ratio (IDR) of 73.6\% [2]. The five widely domesticated species planted as annual crops are capsicum annuum, capsicum frutescense, capsicum chinense, capsicum baccatum, and capsicum pubescence [3], plagues and diseases easily infect these plants. The effects of the infection are a significant reduction in chilli production and deterioration of fruit quality, resulting in low returns for farmers [4]. According to [5], chilli plant diseases are mainly due to the infection caused by pathogenic microbes, namely fungus, bacteria, and viruses. The infection is visible but needs to be examined closely and adequately controlled to avoid the massive diseases spread on the farm. The conventional way of detecting and classifying plant diseases is time-consuming, and automatic detection and classification approaches have been introduced to tackle this problem. In the late 1990s, conventional computer vision 
techniques were used to resolve chilli plant diseases' automatic detection and identification [6]. The major weakness of the traditional computer vision technique was that it was only proven successful on simpler and controlled setups but struggled as the operational conditionschanged [7].

As the years passed, automatic detection and classification of plant diseases utilizing image processing and deep learning approaches have received significant consideration among the experts of the subject. Deep learning is a branch of artificial intelligence that allows machines to perform impressive recognition, prediction, and filtration [8]. Many practical and reliable deep learning algorithms for plant disease classification [9]-[13]. Typically, the classification is performed according to the infected leaf shape and a detectable change in the leaf colour caused by the fungus, bacteria, and virus infection. The application of the transfer learning approach for deep learning has received significant attention. Transfer learning has emerged as a powerful technique whereby the knowledge gained from the larger dataset is transferred to the new dataset [14], [15]. In scenarios within sufficient training data, this technique is beneficial, as presented in research by [16]. In transfer learning, pre-trained models are generally trained on a large scale, such as ImageNet that contains millions of actual images. The advantage is that the learned features are transferred by the weights and the architecture obtained from these models [17]. Inspired by these findings, the performance of the pre-trained model of VGG16, InceptionV3, and EfficientNetB0 in classifying chilli plant disease images captured under an uncontrolled environment with various imaging conditions and a small dataset is studied. This paper shows the performance of these models for classifying highly complex chilli plant diseases images. The findings in this paper will create more opportunities for developing more accurate classifiers in the future. This is because the existing studies have only shown less than $90 \%$ accuracy on a particular type of chilli disease [18], [19]. This paper is organized as follows. Section 2 describes chilli plant disease taxonomy, and section 3 provides the architectures of the used deep learning methods, materials, methods, and experimental setup, and section 4 discusses the results. Finally, the paper is concluded in section 5 .

\section{TAXONOMY OF CHILI DISEASES}

Chilli is a type of plant that can be easily affected by fungi, bacteria, viruses, and pests. Besides, climate changes and the risk of a resistance breakdown can also affect the durability of disease resistance. The example of the fungi, bacteria, viruses, and pests commonly affected by chilli plants [5] are summarized in Figure 1.

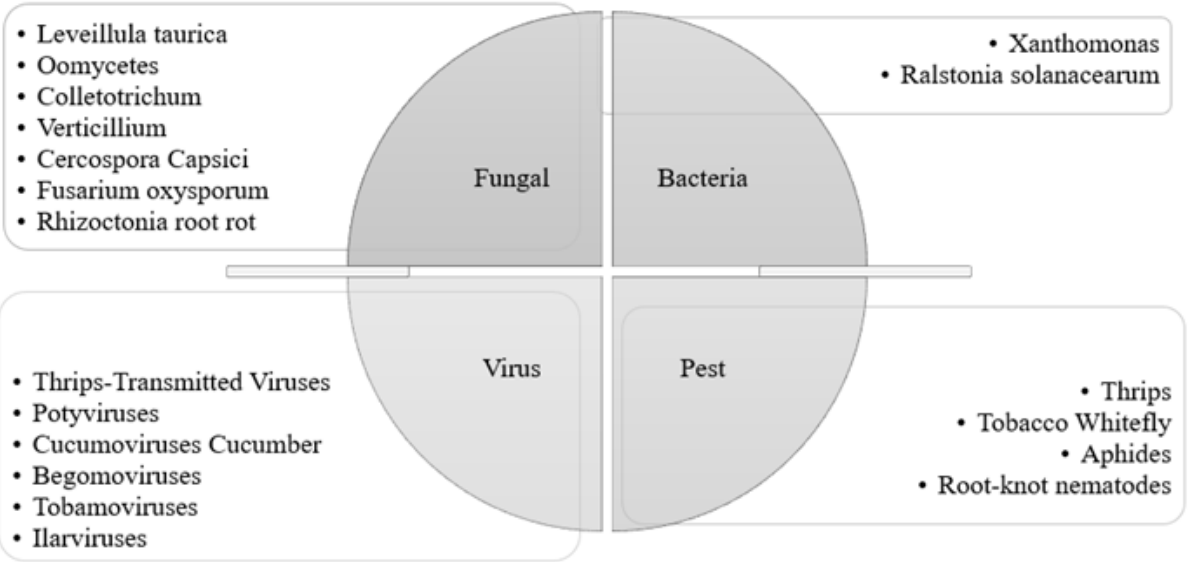

Figure 1. Taxonomy of chilli plant diseases according to [5]

In this study, three types of diseasewere considered: the bacterial spot, upward curling and mosaic/mottling, as shown in Figure 2(a), Figure 2(b), and Figure 2(c), respectively. The bacterial spot is the small black spots of water-soaked on the leaves and gradually browning, coalesce, rugged and cracked. It is mainly due to the pathogen that is known as xanthomonas. The upward curling disease is caused by Begomovirus transmitted by Bemisia whiteflies that caused yellowing of veins and reduced leaf size. The mosaic disease caused the leaves to be yellowed and narrow, which is transmitted mainly by greenfly aphids. 


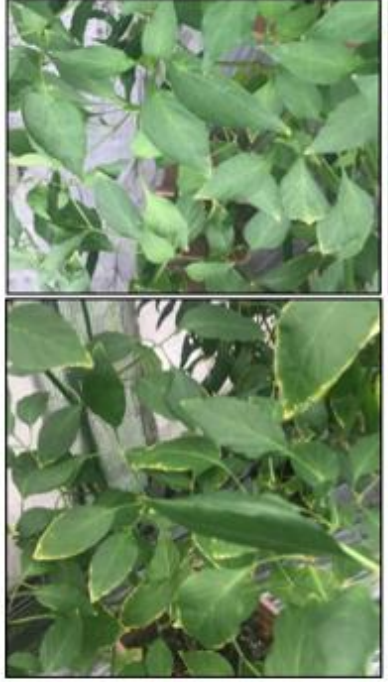

(a)

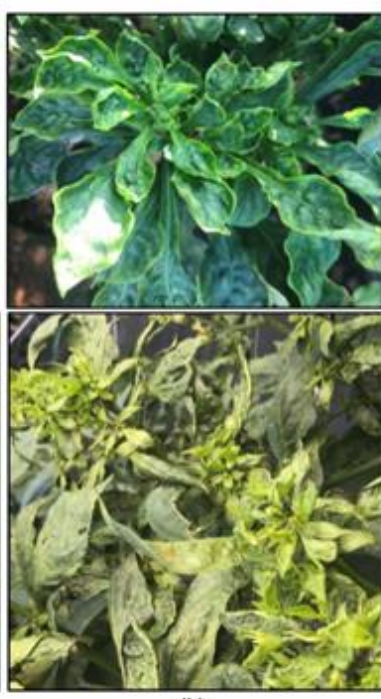

(b)

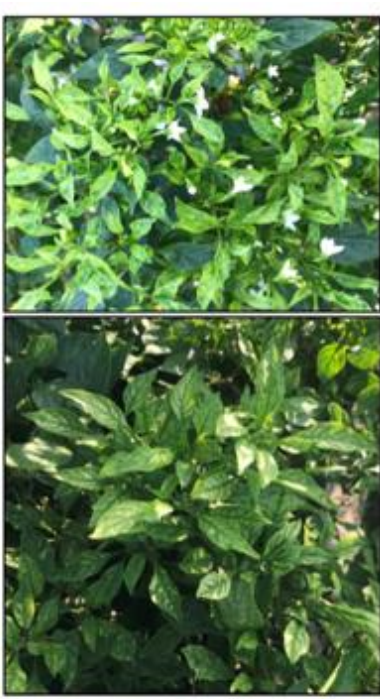

(c)

Figure 2. Samples of chili plant diseases image used in the experiments, (a) bacterial spot, (b) upward curling, and (c) mosaic/mottling

\section{MATERIAL AND METHODS}

\subsection{Chili plant disease dataset}

The dataset used in this study consists of 3,000 images of capsicum annuum L. plants and annotated into three classes of chilli leaves diseases: namely the upward curling, mosaic/mottling and bacterial spot. The images were self-collected under an uncontrolled environment and various illuminations, views, and distances to reflect the real-life scenarios. The images were collected from three different field environments located at Sijangkang Selangor; Community Urban Farm, Bukit Rimau, Selangor, and a greenhouse at the Faculty of Engineering, Universiti Putra Malaysia (UPM) Selangor. The images were captured using Apple iPhone 7 with the dimension of resolution $3024 \times 4032$ and Asus Zenfone 2 with the dimension of resolution $2304 \times 4096$. These images were cropped, resized and flipped manually using Microsoft Photos at the initial stage to reduce the background clutter and occlusion issues. Data annotation was done by consulting the experts at the farms and cross-checking with the related published papers. For each disease, 800 and 200 images were used for training and testing, respectively.

\subsection{Pretrained DCNN model and parameters}

In this study, the performance of VGG16, Inception V3 and EfficientNet B0 in classifying chilli plant diseases from complex images was compared. These models were selected for their outstanding performance when classified the plant disease images from the ImageNet dataset [20]. The VGG16, as illustrated in Figure 3 [21], used a recommended default input image size of $224 \times 224 \times 3$ and 13 convolutional layers with a rectified linear unit (ReLU) activation function. The convolutional layers were fed into a max pooling, three fully connected (FC) layers and a Softmax function at the end of the architecture. The last FC layers were replaced by three channels for this study, indicating the three classes of chilli plant diseases understudy.

Meanwhile, InceptionV3 [22] has 42 total deep network layers with a grid size-reduction block between the inception modules blocks and one auxiliary classifier at the third concatenated trunk, as shown in Figure 4. The recommended size of the input image for this model is $299 \times 299 \times 3$, and five convolutional layers process the input image with two max-pooling layers at the first stage. Then, a series of inception modules process the input before finally performing classification using a fully connected layer and a Softmax function. EfficientNet [20] is a convolutional neural network architecture with a compound scaling method that uniformly scales all depth, width, and resolution dimensions to pursue better accuracy and efficiency. Currently, there are seven versions of EfficientNet networks, in which each version is the upgraded version of the previous, which is scaled from the EfficientNetB0 baseline using different compound coefficients. The EfficientNetB0 network consists of a convolutional layer, several mobile inverted bottleneck convolutional (MBConv) layersand optimization layers, as shown in Figure 5. The recommended size of the input image for this model is $224 \times 224 \times 3$. 


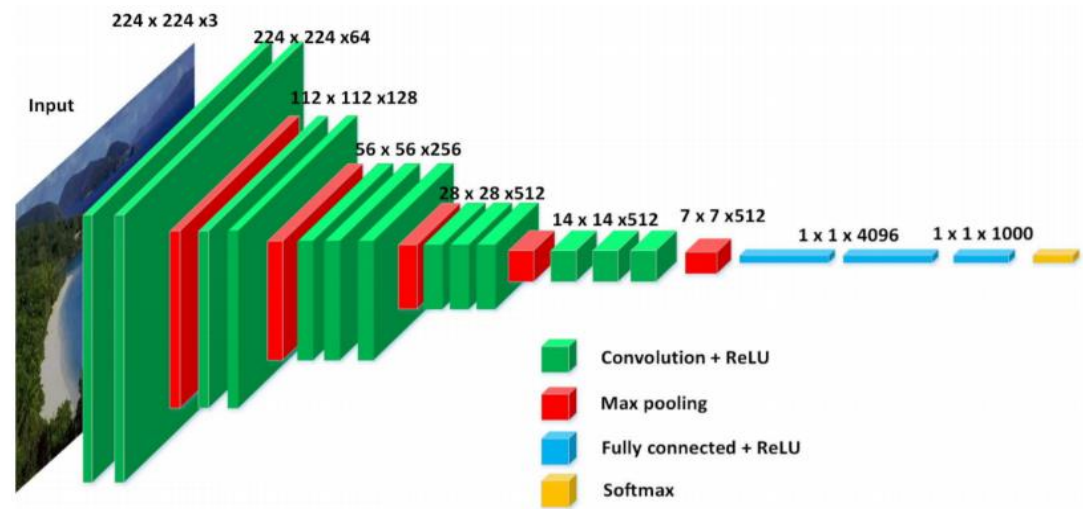

Figure 3. The architecture of the VGG16 network [21]

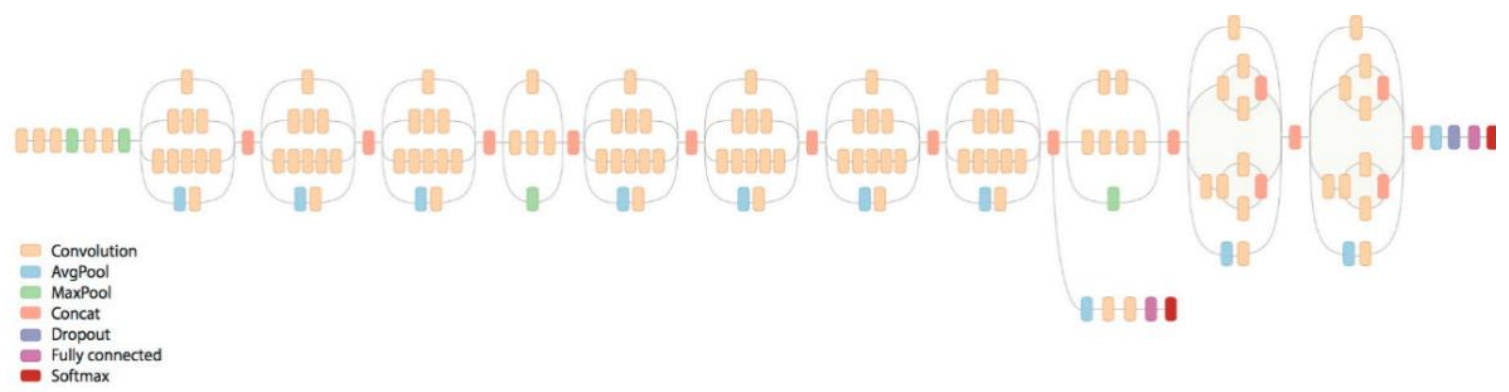

Figure 4. The architecture of InceptionV3 network [23]

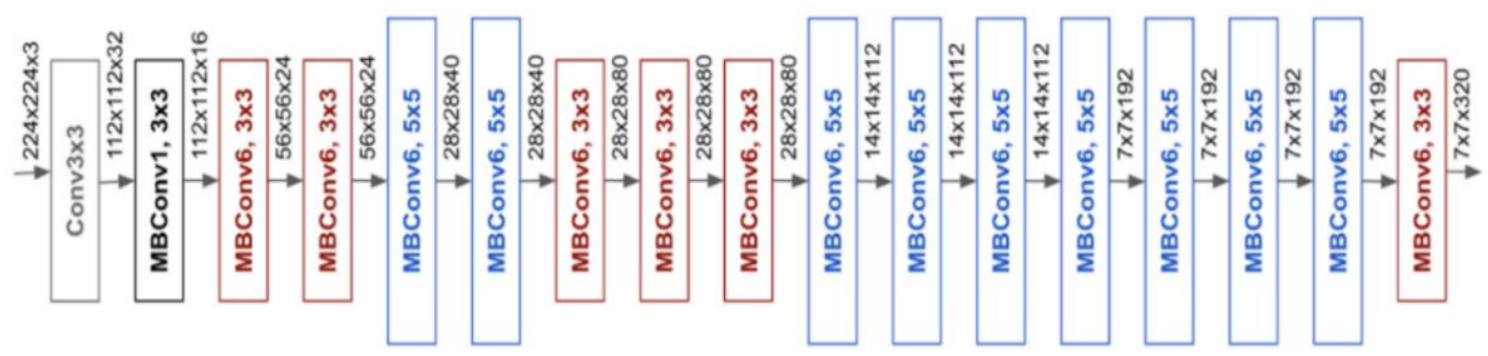

Figure 5. The architecture of EfficientNetB0 network [23]

\subsection{Experimental setup}

The experiment was conducted on a 64-bit operating system, an x64-based processor running on Intel(R) Core (TM) i5-10200H CPU @ 2.40 GHz with NVIDIA GeForce GTX 1650 and 8 GB RAM. All deep learning models were compiled with GPU support. The proposed chilli plant disease classification is shown in Figure 6. The filters, feature maps, pooling layers and hyperparameters of the VGG16, InceptionV3 and EfficientNetB0 models remain the same structure, as obtained from Keras Applications API with ImageNet [24]. Nevertheless, a combination of fully connected layers and Softmax activation was applied. This part has been converted into three outputs representing the chilli plant disease classes (upward curling, bacterial spot and mosaic/mottling). All pre-trained models were set to frozen layers to avoid Keras from updating their weights during the training. Other settings include batch normalization and batch size. Based on [25], each pixel value of the images was divided by 255 for batch normalization, and the selected batch size was 32 . Batch normalization could overcome the problem of internal covariate shift, which can impede the training of deep neural networks. Stochastic gradient descent (SGD) was used as the optimizer due to its high performance [26], while the learning rate of 0.0001 was adopted based on [16]. The epoch is set to 50, and the selectionis based on several trials, such as 10,30, 50 and 100 epochs. The resultshave shown that 50 epochs have produced high accuracy and better processing stability. 
The input images were divided into two sets, $80 \%$ for training and $20 \%$ for testing, as recommended by [27]. The images were resized according to the model's default size, $224 \times 224$ pixels for VGG16 and EfficientNetB0, and 299×299 pixels for Inception V3. The methods were trained with two training sets, where the first set consists of original images and the second setconsists of augmented images. Both sets consist of the same amount of images that is 2400 images. In the second training set, the images were sheared at an angle of 0.2 degrees, zoomed at 0.2 magnification and horizontal flipped using Imagedatagenerator in Kerasapplication. Image data generator works randomly in real-time, with the number of images remaining the same. The augmentation parameters selection was decided based on the observation from a few trials, where the features of the disease can be visualized using these parameters.

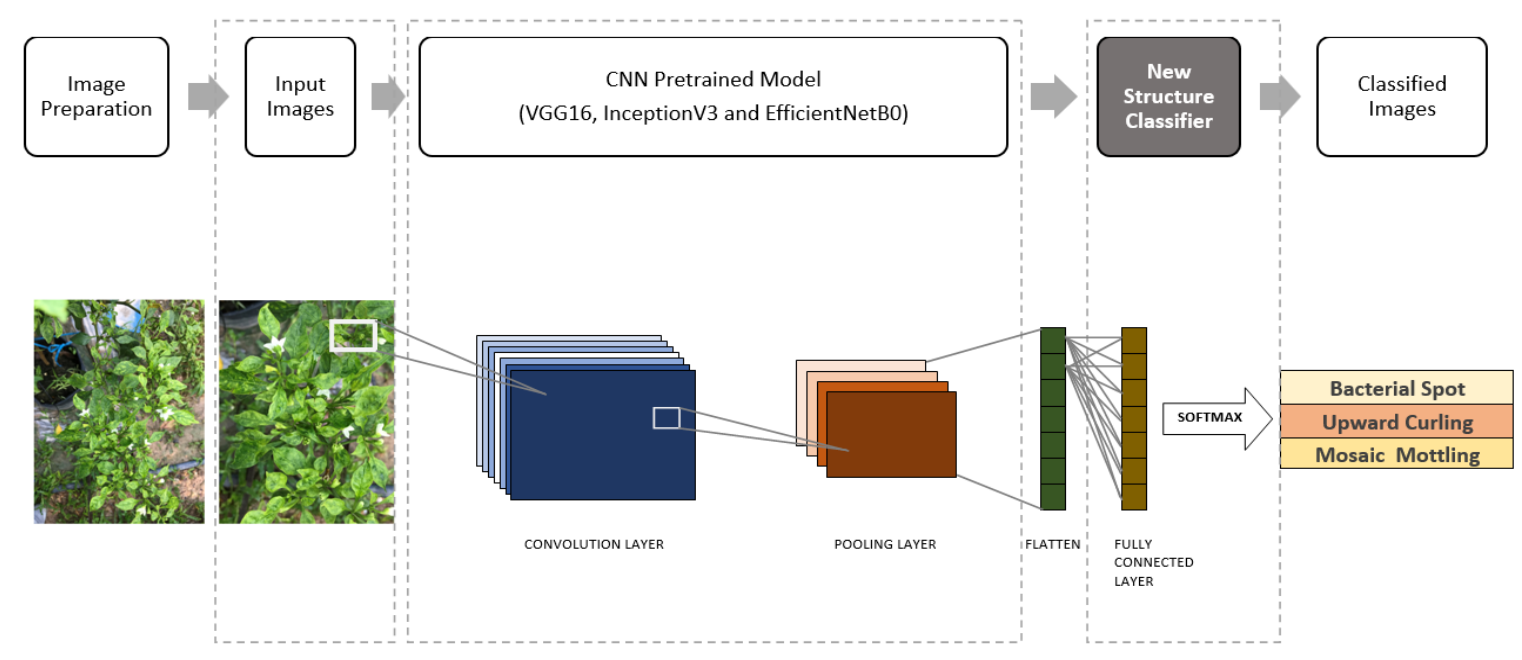

Figure 6. The proposed chili plant disease classification framework

\section{RESULTS AND DISCUSSION}

The performance of the selected deep learning algorithms was evaluated based on accuracy, recall, precision, and F1-score. Accuracy is the number of correctly identified samples, and recall is the number of positive samples that are accurately identified. Meanwhile, precision is the measurement of accurately identified samples among all the true samples, andthe F1-scorerepresents a harmonic mean between sensitivity and precision. The experiments were conducted on two datasets, where the first dataset consist of original images and augmented images in the second dataset. The results in Figure 7(a), Figure 7(b) and Figure 7(c) show that EfficientNetB0 outperformed VGG16 and InceptionV3, but in Figure 8(a), Figure 8(b) and Figure 8(c), it is shown that InceptionV3 outperformed VGG16 and EfficientNetB0. It is also observed that for both cases, VGG16 and InceptionV3 required less computation time compared to EfficientNetB0 to reach above $90 \%$.

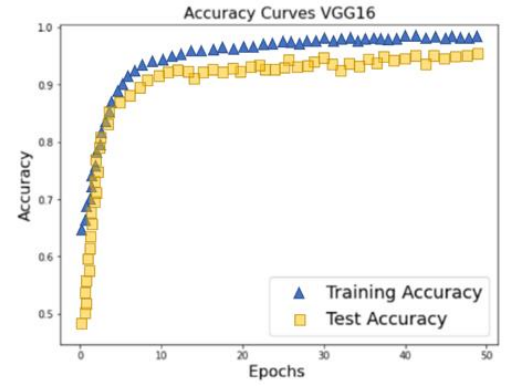

(a)

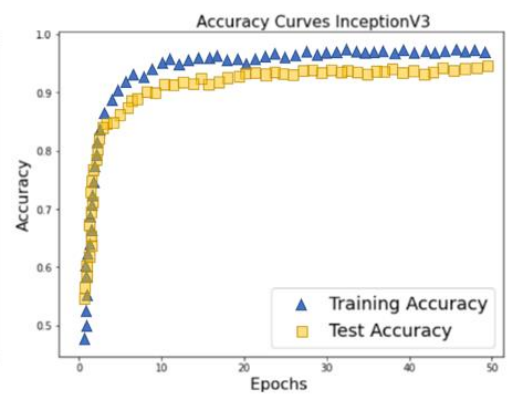

(b)

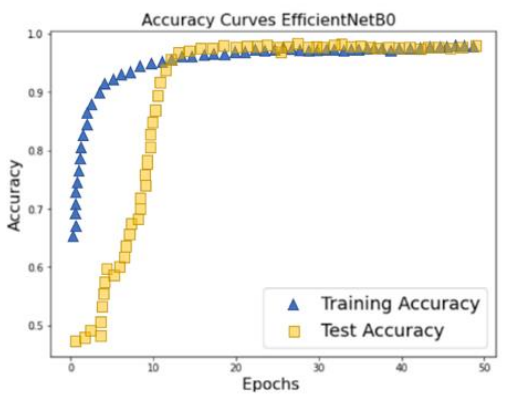

(c)

Figure 7. The accuracy produced by (a) VGG16, (b) InceptionV3, and (c) EfficientNetB0 using original images for training 


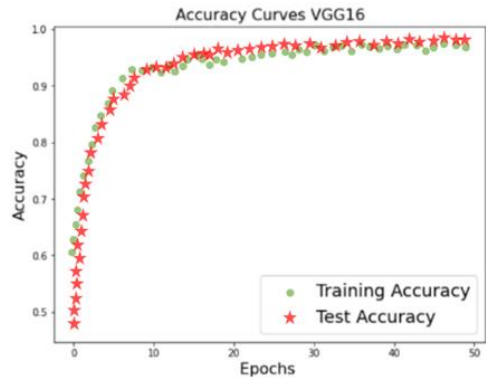

(a)

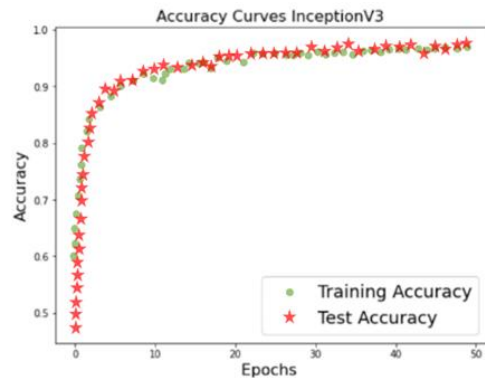

(b)

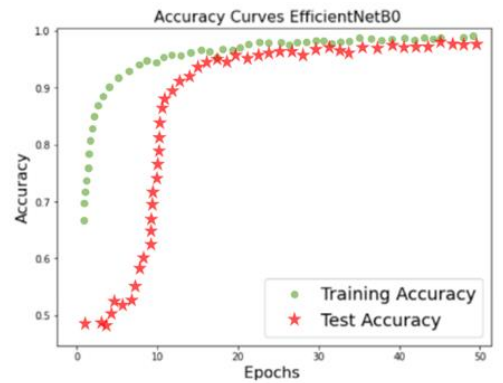

(c)

Figure 8. The accuracy produced by (a) VGG16, (b) InceptionV3, and (c) EfficientNetB0 using augmented images for training

Nevertheless, the accuracy of models trained by original images did not differ much when trained on augmented images. At the 50 epochs, it is shown that the InceptionV3 has produced the highest accuracy of $98.83 \%$ on augmented images while EfficientNetB0 highest accuracy of $97.67 \%$ on original images, respectively. The obtained accuracy, precision, recall, and F1-score for the VGG16, InceptionV3 and EfficientNetB0 are shown in Table 1. It is shown that the performance of EfficientNetB0 and InceptionV3 is approximately similar for both cases, that is, when original or augmented images train the models. However, EfficientNetB0 has better performance when trained by original images, but opposite to InceptionV3, the model performed better when trained by augmented images.

The summary of the classification results for each disease, namely upward curling (UC), mosaic/mottling (M) and bacterial spot (BS) diseases, are shown in the confusion matrix in Table 2 . It is demonstrated that InceptionV3 has produced the highest true positivevalue when classifyingbacterial spots for both cases but the mosaic/mottling, the InceptionV3 model, has the highest true positive value when trained by augmented images. Nevertheless, the true positive value of the InceptionV3 model is lower than the true positive value of EfficientNetB0 when trained by original images. All three methods have equivalent performance when classifying the upward curling disease. It is also shown that the models have difficulty classifying the mosaic/mottling disease, as the produced true positive value is the lowest compared to other diseases. The complex features of the mosaic/mottling disease are approximately similar to the upward curling disease that caused the methods to misclassify.

Table 1. The VGG16, InceptionV3 and EfficientNetB0 testing performance

\begin{tabular}{cccccc}
\hline Training Set & Models & Accuracy (\%) & Precision (\%) & Recall (\%) & F1 Score \\
\hline First set (original images) & VGG16 & 95.17 & 95.00 & 95.00 & 0.95 \\
& InceptionV3 & 97.50 & 97.00 & 97.00 & 0.97 \\
& EfficientNetB0 & $\mathbf{9 7 . 6 7}$ & $\mathbf{9 8 . 0 0}$ & $\mathbf{9 8 . 0 0}$ & $\mathbf{0 . 9 8}$ \\
Second set (augmented images) & VGG16 & 95.83 & 96.00 & 96.00 & 0.96 \\
& InceptionV3 & $\mathbf{9 8 . 8 3}$ & $\mathbf{9 9 . 0 0}$ & $\mathbf{9 9 . 0 0}$ & $\mathbf{0 . 9 9}$ \\
& EfficientNetB0 & 96.83 & 97.00 & 97.00 & 0.97 \\
\hline
\end{tabular}

Table 2. Confusion Matrix of the methods that were trained using original and augmented images

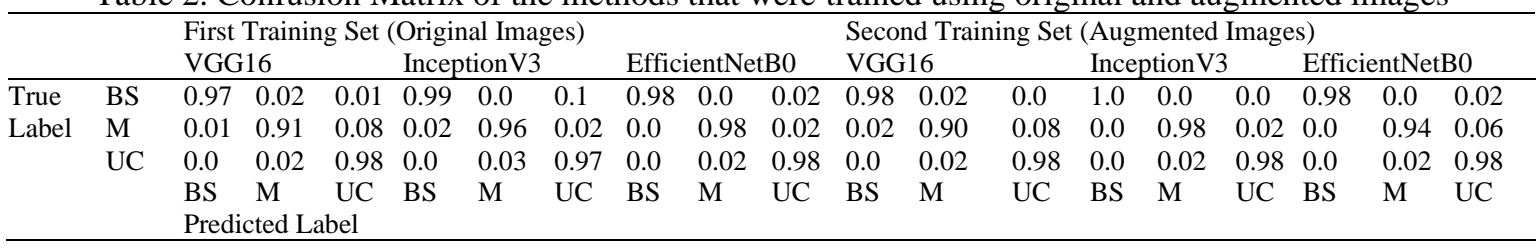

\section{CONCLUSION AND FUTURE WORKS}

The efficacy of deep learning algorithms, namely VGG16, Inception V3 and EfficientNet B0, methods in classifying three types of chilli plant diseases, namely upward curling, mosaic/mottling and bacterial spot from a dataset that consists of 3,000 images in an uncontrolled condition and under various imaging conditions is demonstrated. The experiment results showed that InceptionV3 has better performance than the VGG16 and EfficientNetB0 in classifying bacterial spot images. Still, all the models have difficulty classifyingthe mosaic/mottling disease due to the complex features of the mosaic/mottling disease that are 
approximately similar to the upward curling disease. All three methods have equivalent performance when classifying the upward curling disease. In conclusion, deep learning algorithms have shown a great potential for classifying chilli plant diseases. The performance algorithms can be further improved by exposing them to high-complexity images and several other types of diseases, which will create more opportunities for developing more advanced classifiers.

\section{ACKNOWLEDGEMENTS}

The authors wish to acknowledge the financial support received from the Fundamental Research Grant Scheme (FRGS), Ministry of Higher Education, with grant number 5540078 (ID: 15948 and reference code: FRGS/1/2018/WAB01/UPM/02/30). The authors would also want to extend appreciation to Mrs. Noor Azlina Abdul Aziz and Cui Hairu for their support in this study.

\section{REFERENCES}

[1] S. Lob, M. N. M. Aris, S. N. M. Sidique, N. F. Ibrahim, and X. Jin, "Growth development and natural infection incidence of tobacco mosaic virus (TMV) on silicon-treated chilli (capsicum annuum L.) cultivated in commercial soil," Malaysian Applied Biology, vol. 46, no. 3, pp. 221-226, 2017.

[2] Department of Statistics Malaysia, "Press Release Supply and Utilization Accounts Selected Agricultural Commodities," Department of Statistics, no. December, pp. 2013-2015, 2021.

[3] A. Norfadzilah, "Screening of Selected Cucumber Mosaic Virus Resistance Chilli Genotypes Obtained from World Vegetable Center for High Yield in Malaysian Condition," 2018.

[4] N. Yusuf, S. M. Fazi, N. A. Ali, and N. I. Fauzi, "Effects of Colletotrichum capsici infection on the growth and antioxidative response on defense mechanisms of Capsicum annuum," 2016.

[5] M. Parisi, D. Alioto, and P. Tripodi, "Overview of biotic stresses in pepper (Capsicum spp.): Sources of genetic resistance, molecular breeding and genomics," International Journal of Molecular Sciences, vol. 21, no. 7, 2020, doi: 10.3390/ijms21072587.

[6] K. Pushpanathan, M. Hanafi, S. Mashohor, and W. F. Fazlil Ilahi, "Machine learning in medicinal plants recognition: a review," Artificial Intelligence Review, vol. 54, no. 1, pp. 305-327, 2021, doi: 10.1007/s10462-020-09847-0.

[7] A. Picon, M. Seitz, A. Alvarez-Gila, P. Mohnke, A. Ortiz-Barredo, and J. Echazarra, "Crop conditional Convolutional Neural Networks for massive multi-crop plant disease classification over cell phone acquired images taken on real field conditions," Computers and Electronics in Agriculture, vol. 167, no. September, p. 105093, 2019, doi: 10.1016/j.compag.2019.105093.

[8] R. I. Hasan, S. M. Yusuf, and L. Alzubaidi, "Review of the state of the art of deep learning for plant diseases: A broad analysis and discussion," Plants, vol. 9, no. 10, pp. 1-25, 2020, doi: 10.3390/plants9101302.

[9] J. Liu and X. Wang, "Plant diseases and pests detection based on deep learning: a review," Plant Methods, vol. 17, no. 1, pp. 118, 2021, doi: 10.1186/s13007-021-00722-9.

[10] Ü. Atila, M. Uçar, K. Akyol, and E. Uçar, "Plant leaf disease classification using EfficientNet deep learning model," Ecological Informatics, vol. 61, no. October 2020, p. 101182, 2021, doi: 10.1016/j.ecoinf.2020.101182.

[11] J. Chen, J. Chen, D. Zhang, Y. Sun, and Y. A. Nanehkaran, "Using deep transfer learning for image-based plant disease identification," Computers and Electronics in Agriculture, vol. 173, no. April, p. 105393, 2020, doi: 10.1016/j.compag.2020.105393.

[12] A. Ramdan, A. Heryana, A. Arisal, R. B. S. Kusumo, and H. F. Pardede, "Transfer Learning and Fine-Tuning for Deep LearningBased Tea Diseases Detection on Small Datasets," Proceeding - 2020 International Conference on Radar, Antenna, Microwave, Electronics and Telecommunications, ICRAMET 2020, pp. 206-211, 2020, doi: 10.1109/ICRAMET51080.2020.9298575.

[13] A. R. Luaibi, T. M. Salman, and A. H. Miry, "Detection of citrus leaf diseases using a deep learning technique," International Journal of Electrical and Computer Engineering, vol. 11, no. 2, pp. 1719-1727, 2021, doi: 10.11591/ijece.v11i2.pp1719-1727.

[14] W. Zhu, B. Braun, L. H. Chiang, and J. A. Romagnoli, "Investigation of transfer learning for image classification and impact on training sample size," Chemometrics and Intelligent Laboratory Systems, vol. 211, no. February, p. 104269, 2021, doi: 10.1016/j.chemolab.2021.104269.

[15] S. Niu, Y. Liu, J. Wang, and H. Song, “A Decade Survey of Transfer Learning (2010-2020)," IEEE Transactions on Artificial Intelligence, vol. 1, no. 2, pp. 151-166, 2021, doi: 10.1109/tai.2021.3054609.

[16] Y. Wu, X. Qin, Y. Pan, and C. Yuan, "Convolution neural network based transfer learning for classification of flowers," 2018 IEEE 3rd International Conference on Signal and Image Processing, ICSIP 2018, pp. 562-566, 2019, doi: 10.1109/SIPROCESS.2018.8600536.

[17] C. Tan, F. Sun, T. Kong, W. Zhang, C. Yang, and C. Liu, "A survey on deep transfer learning," Lecture Notes in Computer Science (including subseries Lecture Notes in Artificial Intelligence and Lecture Notes in Bioinformatics), vol. 11141 LNCS, pp. 270-279, 2018, doi: 10.1007/978-3-030-01424-7 27.

[18] T. Purwaningsih, I. A. Anjani, and P. B. Utami, "Convolutional Neural Networks Implementation for Chili Classification," Proceeding - 2018 International Symposium on Advanced Intelligent Informatics: Revolutionize Intelligent Informatics Spectrum for Humanity, SAIN 2018, pp. 190-194, 2019, doi: 10.1109/SAIN.2018.8673373.

[19] A. R. Bahtiar, Pranowo, A. J. Santoso, and J. Juhariah, "Deep Learning Detected Nutrient Deficiency in Chili Plant," 2020 8th International Conference on Information and Communication Technology, ICoICT 2020, pp. 5-8, 2020, doi: 10.1109/ICoICT49345.2020.9166224.

[20] M. Tan and Q. V. Le, "EfficientNet: Rethinking model scaling for convolutional neural networks," 36th International Conference on Machine Learning, ICML 2019, vol. 2019-June, pp. 10691-10700, 2019.

[21] F. Liu, Y. Wang, F. C. Wang, Y. Z. Zhang, and J. Lin, "Intelligent and secure content-based image retrieval for mobile users," IEEE Access, vol. 7, pp. 119209-119222, 2019, doi: 10.1109/ACCESS.2019.2935222.

[22] C. Szegedy, V. Vanhoucke, S. Ioffe, J. Shlens, and Z. Wojna, "Rethinking the Inception Architecture for Computer Vision," Proceedings of the IEEE Computer Society Conference on Computer Vision and Pattern Recognition, vol. 2016-December, pp. 2818-2826, 2016, doi: 10.1109/CVPR.2016.308. 
[23] Y. Bazi, M. M. A. Rahhal, H. Alhichri, and N. Alajlan, "Simple yet effective fine-tuning of deep cnns using an auxiliary classification loss for remote sensing scene classification," Remote Sensing, vol. 11, no. 24, 2019, doi: 10.3390/rs11242908.

[24] A. Krizhevsky, I. Sutskever, and G. E. Hinton, "ImageNet classification with deep convolutional neural networks," Communications of the ACM, vol. 60, no. 6, pp. 84-90, 2017, doi: 10.1145/3065386.

[25] D. Masters and C. Luschi, "Revisiting Small Batch Training for Deep Neural Networks," pp. 1-18, 2018, [Online]. Available: http://arxiv.org/abs/1804.07612.

[26] V. Maeda-Gutiérrez et al., "Comparison of convolutional neural network architectures for classification of tomato plant diseases," Applied Sciences (Switzerland), vol. 10, no. 4, 2020, doi: 10.3390/app10041245.

[27] S. P. Mohanty, D. P. Hughes, and M. Salathé, "Using deep learning for image-based plant disease detection," Frontiers in Plant Science, vol. 7, no. September, 2016, doi: 10.3389/fpls.2016.01419.

\section{BIOGRAPHIES OF AUTHORS}

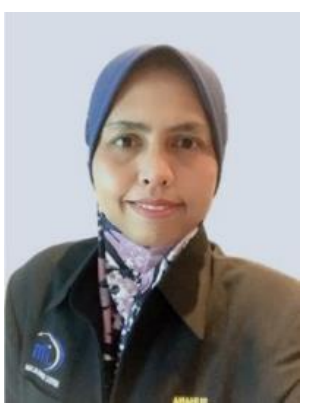

Suhana Rozlan (D) 8I SC P received the Bachelor of Electronic Engineering from Universiti Tun Hussein Onn Malaysia (UTHM), in 2003. She is currently pursuing the Master of Communication EngineeringatUniversiti Putra Malaysia (UPM). Her research interest includes computer vision applications in agriculture, using deep learning methods to study plant leaf disease detection in complex backgrounds. She can be contacted at email: gs55752@student.upm.edu.my.

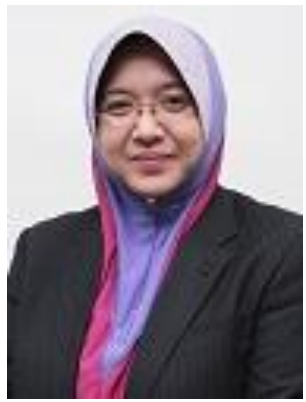

Marsyita Hanafi (D) 8D SC P is currently a Senior Lecturer at the Department of Computer and Communication Systems, Faculty of Engineering, Universiti Putra Malaysia (UPM). She received her Ph.D. in Image Processing and Artificial Intelligence from Imperial College London, UK, in 2012. Her research interests are image processing and artificial intelligence that include autonomous vehicles, precision agriculture, biometric, and IoT-based intelligent monitoring systems. She is actively involved with multi-discipline research studying AI and image processing applications with the Faculty of Agriculture, Faculty of Medicine, and Faculty of Education. She has been involved with IEEE as a member since 2012. She is the principal investigator and collaborator for projects under the local funding bodies; namely the Malaysian Ministry of Higher Education (MoHE), and Research University Grant Scheme (RUGS) (now known as Putra Initiative Grant) UPM. She can be contacted at email: marsyita@upm.edu.my. 